\title{
Insufficient Number of Beats for Complete ECG Evaluation
}

National Cancer Institute

\section{Source}

National Cancer Institute. Insufficient Number of Beats for Complete ECG Evaluation.

NCI Thesaurus. Code C114177.

An electrocardiog raphic recording in which fewer than the required number of cardiac complexes are available for measurement and/or interpretation due to a shorter than planned recording or due to cardiac complexes which are present but are not suitable for measurement and/or interpretation. (CDISC) 